\title{
Des sens au sens. Littérature \& morale de Molière à Voltaire, études réunies et présentées par Jacques Wagner
}

\section{Bianca Gai}

\section{(2) OpenEdition Journals}

Edizione digitale

URL: http://journals.openedition.org/studifrancesi/7921

DOI: 10.4000/studifrancesi.7921

ISSN: 2421-5856

\section{Editore}

Rosenberg \& Sellier

\section{Edizione cartacea}

Data di pubblicazione: 1 juillet 2009

Paginazione: 394-396

ISSN: 0039-2944

\section{Notizia bibliografica digitale}

Bianca Gai, «Des sens au sens. Littérature \& morale de Molière à Voltaire, études réunies et présentées par Jacques Wagner», Studi Francesi [Online], 158 (LIII | II) | 2009, online dal 30 novembre 2015, consultato il 08 janvier 2021. URL: http://journals.openedition.org/studifrancesi/7921 ; DOI: https:// doi.org/ERREUR PDO dans /localdata/www-bin/Core/Core/Db/Db.class.php L.34 : SQLSTATE[HY000] [2006] MySQL server has gone away

Questo documento è stato generato automaticamente il 8 janvier 2021.

\section{(†)

Studi Francesi è distribuita con Licenza Creative Commons Attribuzione - Non commerciale - Non opere derivate 4.0 Internazionale. 


\title{
Des sens au sens. Littérature \& morale de Molière à Voltaire, études réunies et présentées par Jacques Wagner
}

\author{
Bianca Gai
}

\section{NOTIZIA}

Des sens au sens. Littérature \& morale de Molière à Voltaire, études réunies et présentées par Jacques WAGNER, Louvain-Paris-Dudley, MA, Peeters, 2007, pp. 200.

1 Il gioco di parole del titolo individua la dicotomia che struttura gli studi proposti, volti a ripercorrere, come illustra la Présentation del curatore Jacques W AGNER, i diversi equilibri che la correlazione problematica fra sensibilità e morale assume lungo l'arco temporale 1660-1778, attraverso esemplificazioni che toccano molteplici generi e forme espressive. Radice comune a tutti gli interventi è la constatazione del netto rovesciamento di valori verificatosi nel rapporto fra "sensi" e "senso" morale, in seguito al passaggio dal classicismo seicentesco all'estetica del xvIII secolo: l'esperienza sensuale non è più sottoposta a strategie di censura nella costituzione della morale, ma anzi diventa dato di partenza per l'affermazione di una nuova volontà "impura", che arriva a destabilizzare la nozione stessa di "senso" morale. A partire da tale ipotesi comune, si delinea lungo il volume una serie di differenti combinazioni nelle modalità di relazione fra le due variabili oggetto di analisi.

2 L'indagine si apre sulla figura del Misanthrope di Molière, di cui Jacques CHUPEAU (Variations sur "Le Misanthrope": Alceste, Philinte et Célimène, ou de l'héroïsme, de l'honnêteté et de la liberté, pp. 13-23) rintraccia le modificazioni di ricezione dal Seicento a oggi. Lo statuto ambivalente del personaggio di Alceste, tra l'eroico e il ridicolo, indica in maniera esemplare una nuova volontà di derisione del pensiero idealizzante, testimoniata in negativo dalla condanna dell'atteggiamento dell'autore nei confronti della virtù del protagonista che sarà avanzata da Fénelon, Rousseau e dagli scrittori 
romantici. Un altro caso di scissione fra ambito sensuale e ambito morale è trattato nel saggio di Hélène CusSAC ( $L a$ volonté et la loi annihilées par le pouvoir des sens dans "Les Illustres Françaises", pp. 25-36), dedicato al romanzo di Robert Challe, in cui si osserva un procedimento di sostituzione delle sensazioni, soprattutto uditive, ai valori morali tradizionali. Udito e vista provocano un indebolimento e una deviazione delle volontà dei personaggi, causandone profonde modificazioni interiori e sconvolgendone così i destini romanzeschi. Il romanzo di Prévost Campagnes philosophiques costituisce invece, come emerge dallo studio di Paul Pelckmans (Une velléité d'Apocalypse, à propos du dénouement des "Campagnes philosophiques", pp. 37-47), un esempio di sintesi fra sensi e virtù. Seppure si assista nell'opera a un frequente rifiuto della validità etica dell'eccesso di passione, che comporta per i personaggi la trasformazione di qualsiasi impegno troppo appassionato in castigo, il Supplément che ne conclude la vicenda riconfigura la morale in un'ottica sensualista, postulando nel presente la coincidenza fra virtù e esistenza felice, in una prospettiva anticipatamente rousseauiana.

Secondo Marie-Paule DE WEERDT-PILORGE (L'écriture de soi dans les mémoires et romansmémoires au XVIII siècle: les années de formation, pp. 51-60), anche La Vie de Marianne teorizza la conciliazione di elemento fisico e morale. L'autrice illustra le tangenze fra il roman-mémoire di Marivaux e i mémoires settecenteschi non fittizi, per quanto concerne le modalità di costruzione dell'identità individuale: i meccanismi di definizione dell'io, per esempio il valore positivo conferito all'amour-propre nell'evoluzione della personalità, evidenziano la nascita di una sensibilità non più definita da un'azione sacrificale di fronte ai fattori istintuali, ma fondata su moventi edonisti e pragmatici.

4 Nel lavoro che segue, Emmanuel Boussuge (L'homme au coeur velu: autour d'une anecdote de la "Satire première". Le heurt de deux sensibilités: Fougeret de Monbron et Diderot, pp. 61-94) focalizza la polarità instaurata, nella Satire première di Diderot, fra la voce narrante e la figura di Fougeret de Monbron, il quale, ispirandosi a concezioni di origine hobbesiana, crea una divaricazione inconciliabile fra piacere e morale, riducendo la portata della virtù a impulso puramente automatico, slegato dalla sensibilità. L'inserzione nella satira di un aneddoto che pone a confronto il trasporto patetico di Diderot davanti a uno spettacolo teatrale e l'imperturbabilità di Fougeret costituisce un contraltare polemico alle certezze del filosofo in merito al carattere universale della sensibilità e al valore dell'esperienza teatrale come momento collettivo unificante, avanzando una poetica dell'insensibilità totalmente inaccettabile nella prospettiva teorica dell'autore. Ancora un accordo fra "senso" e "sensi" si stabilisce nei racconti di Marmontel secondo Anne-Marie CABANAT (À l'école du bonheur, quelle place pour le désir?, pp. 95-107), che ne ripercorre le varie sfumature delle trame d'amore, sullo sfondo della filosofia socratica del Convivio platonico, corretta però da una visione epicurea che confida nella saggezza e misura del piacere. Si verifica così una reintegrazione della trasgressione sensuale sulla via della virtù, cui si giunge tuttavia senza una repressione delle inclinazioni sensuali di partenza.

Jacques SPICA (Nature humaine et humaine nature, pp. 109-140) riconosce una simile strategia di compromesso fra dimensione morale e sensuale nei racconti di Voltaire. Il punto di partenza teoretico è un'articolazione dell'universo secondo una scala di esseri, accostati per giustapposizione. Il regno minerale si differenzia da quello animale per la presenza dell'istinto, mentre la comparsa della morale, originatasi a partire dal sentimento di 'bienveillance naturelle' che lega tutti gli uomini, distingue la sfera umana da quella animale. L'uomo non può prescindere dal proprio sostrato animale, 
ma può ricostruire in perpetuo nel mondo la propria umanità e costituirsi come fine della propria azione. Ancora su Voltaire si concentra il saggio di Jacques WAGNER (Des choses, des hommes et des insectes dans les cuvres philosophiques de Voltaire (1734-1769) ou les prémices d'une esthétique du quotidien et du familier, pp. 143-160), che illustra la tendenza dell'autore a costellare la propria scrittura filosofica di una grande quantità di oggetti materiali, secondo un meccanismo contrario all'estetica idealizzante del classicismo, di cui pure Voltaire è un convinto seguace, in cui gli oggetti non trovano al contrario alcuno spazio di comparsa. Dalle Lettres philosophiques al Dictionnaire philosophique, $\mathrm{i}$ riferimenti al mondo materiale si moltiplicano e si differenziano, passando da una valenza ancora legata alla tradizione satirica e moralistica, a un'estetica del quotidiano che esplicita una morale tesa a rivalutare i bisogni primordiali dell'uomo, mediante l'eliminazione di qualsiasi valenza spiritualista del linguaggio.

Una incongruente convivenza fra valori sensuali e moralizzanti è ravvisata da Mladen Kozul ( $L a$ séduction critique: la lecture du roman entre la théologie et le libertinage, pp. 161-183) nella critica settecentesca al romanzo, che anche nei casi di più forte intento censorio integra al suo interno il discorso romanzesco, mimandone i procedimenti nel momento stesso in cui ne commenta il funzionamento. Si instaura una singolare aporia: per convincere in merito all'influenza negativa del romanzo sull'immaginazione del lettore è necessario argomentarne l'efficacia estetica, talvolta anche mediante la messa in scena delle modificazioni fisiologiche inerenti all'atto della lettura, con un rovesciamento che arriva a porre il critico in una posizione voyeuristica analoga a quella assunta, per esempio, dal narratore dei romanzi libertini.

7 Chiude la miscellanea l'intervento di Daniel Dumouchel (Les paradoxes du «sens» esthétique chez Diderot, pp. 185-200), che illustra lo statuto paradossale della teoria estetica di Diderot, estrema esemplificazione della tensione contraddittoria fra sensibilità e moralità che anima tutto il Settecento. L'autore dimostra come l'estetica del philosophe si possa considerare irriducibile al suo materialismo, e anzi si avvicini piuttosto a posizioni di tipo razionalista. Il giudizio sul bello esibisce alcune somiglianze con i meccanismi della percezione sensibile, ma non si riduce al senso, anzi a partire dalle prime opere di Diderot si rileva una forte critica dei limiti della sensibilità, che contraddice tuttavia la concezione diderotiana del patetico come mezzo per veicolare effetti morali. 